\title{
Design and fabrication of auxetic PCL nanofiber membranes for biomedical applications
}

\author{
Sukhwinder K. Bhullar ${ }^{\mathrm{a}, \mathrm{b}, *}$, Deepti Rana ${ }^{\mathrm{c}}$, Huseyin Lekesiz ${ }^{\mathrm{a}}$, Ayse Celik Bedeloglu ${ }^{\mathrm{d}}$, \\ Junghyuk Ko ${ }^{\mathrm{b}}$, Yonghyun Cho ${ }^{\mathrm{b}}$, Zeynep Aytac ${ }^{\mathrm{e}}$, Tamer Uyar ${ }^{\mathrm{e}}$, Martin Jun ${ }^{\mathrm{b}}$, \\ Murugan Ramalingam ${ }^{\mathrm{c}, \mathrm{f}_{\text {,** }}}$ \\ a Department of Mechanical Engineering, Bursa Technical University, Bursa, Turkey \\ b Department of Mechanical Engineering, University of Victoria, Victoria, BC, Canada \\ c Centre for Stem Cell Research (CSCR), A unit of Institute for Stem Cell Biology and Regenerative Medicine - Bengaluru, Christian Medical College Campus, Vellore \\ 632002, India \\ d Department of Fiber and Polymer Engineering, Bursa Technical University, Bursa, Turkey \\ e Institute of Materials Science \& Nanotechnology, UNAM-National Nanotechnology Research Center, Bilkent University, 06800 Ankara, Turkey \\ ${ }^{f}$ WPI-Advanced Institute for Materials Research, Tohoku University, Sendai 980-8577, Japan
}

\section{A R T I C L E I N F O}

\section{Keywords:}

Electrospinning

Auxetic nanofiber membranes

Poly ( $\varepsilon$-caprolactone)

Mechanical behavior

Biomedical applications

\begin{abstract}
A B S T R A C T
The main objective of this study was to fabricate poly ( $\varepsilon$-caprolactone) (PCL)-based auxetic nanofiber membranes and characterize them for their mechanical and physicochemical properties. As a first step, the PCL nanofibers were fabricated by electrospinning with two different thicknesses of $40 \mu \mathrm{m}$ (called PCL thin membrane) and $180 \mu \mathrm{m}$ (called PCL thick membrane). In the second step, they were tailored into auxetic patterns using femtosecond laser cut technique. The physicochemical and mechanical properties of the auxetic nanofiber membranes were studied and compared with the conventional electrospun PCL nanofibers (non-auxetic nanofiber membranes) as a control. The results showed that there were no significant changes observed among them in terms of their chemical functionality and thermal property. However, there was a notable difference observed in the mechanical properties. For instance, the thin auxetic nanofiber membrane showed the magnitude of elongation almost ten times higher than the control, which clearly demonstrates the high flexibility of auxetic nanofiber membranes. This is because that the auxetic nanofiber membranes have lesser rigidity than the control nanofibers under the same load which could be due to the rotational motion of the auxetic structures. The major finding of this study is that the auxetic PCL nanofiber membranes are highly flexible (10-fold higher elongation capacity than the conventional PCL nanofibers) and have tunable mechanical properties. Therefore, the auxetic PCL nanofiber membranes may serve as a potent material in various biomedical applications, in particular, tissue engineering where scaffolds with mechanical cues play a major role.
\end{abstract}

\section{Introduction}

The auxetic or negative Poisson's ratio materials are of great interest for various biomedical applications, in particular, tissue engineering as scaffolds owing to their unique mechanical and cell responsive behavior. Scaffold-based tissue engineering involves culturing of isolated cells from the patient or donor into a scaffolding system that support the growth and function of the isolated cells into a specific tissue which could be grafted back to the defective site of the patient where tissue regeneration is required [1]. The key components which determine the success of tissue engineering include cells, engineered matrices (also called scaffolds) and cell-matrix interactions. The scaffold plays a key role in tissue engineering by providing a structural support for the cells to accommodate and guide their growth in three-dimensional (3D) space into a specific tissue or organ [2]. Polymers, which are biocompatible and in the form of nanofibers, are widely used as a scaffolding system for tissue engineering applications owing to their structural and functional properties [3-6]. The polymeric nanofiber scaffolds offer large surface area-to-volume ratio and high porosity, which favors in modulating cellular fate and function during tissue

\footnotetext{
* Correspondence to: S.K. Bhullar, Department of Mechanical Engineering, Bursa Technical University, Bursa, Turkey.

*** Correspondence to: M. Ramalingam, Centre for Stem Cell Research (CSCR), A unit of Institute for Stem Cell Biology and Regenerative Medicine - Bengaluru, Christian Medical College Campus, Vellore 632002, India.

E-mail addresses: kaur.bhullar@btu.edu.tr, sbhullar@uvic.ca (S.K. Bhullar),rmurug2000@gmail.com (M. Ramalingam).
} 
(A)

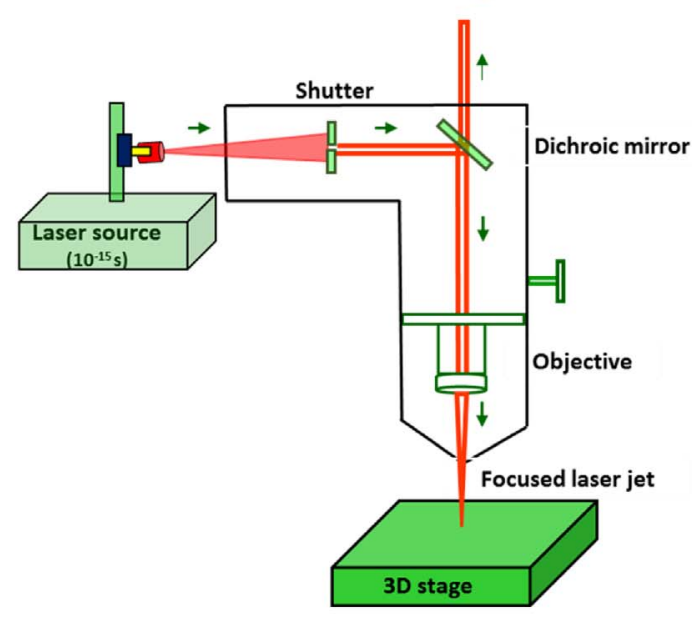

(B)

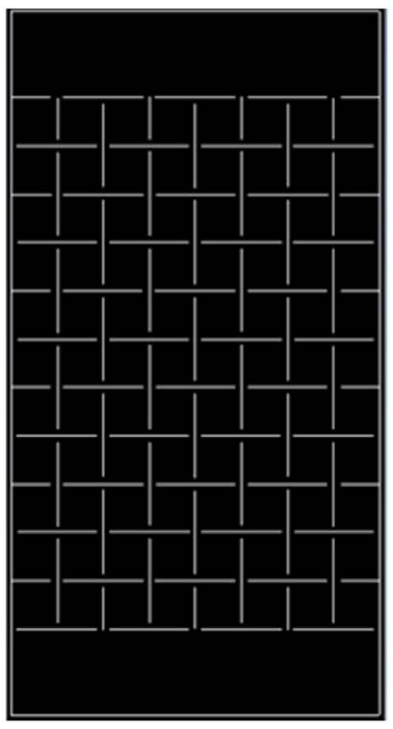

Fig. 1. (A) Schematic diagram of laser micromachining used to tailor auxetic patterns on nanofibers (B) Sketched auxetic pattern for making auxetic nanofiber membrane using Solidworks software. engineering [1,7-11]. However, most of the conventional polymer nanofiber scaffolds are limited by inadequate mechanical properties such as flexibility, elongation, etc. and poor cellular infiltration in $3 \mathrm{D}$, which are highly essential characteristics for tissue engineering applications where mechanical cues play a major role in controlling cell fate and function $[12,13]$.

Therefore, to address the above limitations, the auxetic nanofiber materials have been introduced in the recent days. The auxetic material possesses adequate cell responsive and excellent mechanical properties such as flexibility, indentation resistance, fracture toughness, resilience, vibration control, shear resistance and deformation mechanism compared to conventional material of the same origin due to their unique structural designed patterns [1,14-18]. The auxetic patterned materials have the ability to undergo wider when stretched, thinner when compressed and behave synclastically on bending [19-21]. Some of the naturally occurring biological auxetic systems are cat skin, cow teat skin, salamander skin, load-bearing cancellous bone from human shins and arterial endothelium tissue [22-25]. Therefore, auxetic materials are attracted for tissue engineering applications in the form of scaffolds, stents, dilators, artificial blood vessels, filters implants and prostheses [26-31].

Auxetic scaffolds have been made by using various polymers such as polyuratehylene, polypropylene, ultra-high-molecular-weight polyethylene, polytetrafluoroethylene, polyurethane and poly( $\varepsilon$-caprolactone) (PCL) for various biomedical applications. However, auxetic polymeric nanofiber scaffolds produced by electrospinning are very limited and the research on this field is still at infancy. The nonwoven nano-and micro-fibrous structures are becoming popular for a wide variety of tissue engineering and other biomedical applications such as biomedical devices, filtration, sensors, optical applications and nanofibers reinforced composites [32,33]. For instance, auxetic scaffolds have been shown to possess improved elastic and other mechanical properties than the conventional scaffolds [34]. Recently, Yan et al. investigated the role of auxetic scaffolds with tunable Poisson's ratio in providing a unique biophysical 3D microenvironment which could influence stem cell fate [1]. The results from this study suggested that tailorable auxetic scaffolds together with elastic modulus and microstructure could modulate the 3D biophysical microenvironment as well as influence stem cells differentiation; thereby making them a potent scaffolding system. Keeping the above points in view, in this study, authors report the preparation and characterization of auxetic PCL nanofiber membranes suitable for various biomedical applications, in particular, tissue engineering. PCL is one of the widely used polymers for fabricating scaffolding systems for use in cell culture and tissue engineering applications.

\section{Materials and method}

\subsection{Materials}

For the fabrication of nanofibers, PCL (relative molecular mass $80,000 \mathrm{~g} / \mathrm{mol}$ ), chloroform and methanol were purchased from Sigma Aldrich, USA. All materials and solvents were used as received condition.

\subsection{Preparation of PCL nanofibers}

The PCL nanofiber membranes were fabricated by a custom-made electrospinning apparatus. In brief, a 10\% PCL solution was made using chloroform and methanol (3:1 volume ratio) mixture as solvent. Subsequently, the PCL solution was loaded into a $5 \mathrm{ml}$ syringe (18 gauge). The syringe tip was vertically oriented $17 \mathrm{~cm}$ above the target (aluminum foil). The flow rate was fixed at $2 \mathrm{ml} / \mathrm{h}$ by using a syringe pump (New Era Pump Systems Inc., USA) with a supply of $16 \mathrm{kV}$ voltage using a high voltage power supply (Gamma High Voltage Research Inc., USA) at $24^{\circ} \mathrm{C}$ and $55 \%$ relative humidity. By varying the electrospinning time, two different thickness of nanofiber membranes were fabricated; namely, thin nanofiber membrane with $40 \mu \mathrm{m}$ thickness (hereafter called as PCL) and thick nanofiber membrane with $180 \mu \mathrm{m}$ thickness (hereafter called as PCLT).

\subsection{Auxetic patterning of PCL nanofibers}

The auxetic patterns were made on two different thicknesses of PCL $(40 \mu \mathrm{m})$ and PCLT $(180 \mu \mathrm{m})$ samples using laser micromachining as shown in Fig. 1(A). The schematic diagram of femtosecond laser system used in tailoring sketched auxetic pattern using Solidworks software have been shown in Fig. 1(B). The titanium sapphire femtosecond laser with $120 \mathrm{fs}$ pulse duration and $1 \mathrm{kHz}$ repetition rate at a central wavelength of $800 \mathrm{~nm}$ was used to ablate the materials in the experiment. The laser beam was guided into microscopic objective lenses and focused by $20 \times$ objective lenses with 0.42 Numerical Aperture (Mitutoyo). An iris diaphragm was used to reduce beam diameter to $3 \mathrm{~mm}$ for thin width of cut. An electronic shutter controlled by computer was used to timely turn on/off the laser beam at desired location on the samples. The structure machined during irradiation of femtosecond pulses can be observed through a CCD camera installed above the dichroic mirror and the spot size of laser beam focused onto the sample 
Table 1

List of samples fabricated for material characterization.

\begin{tabular}{lll}
\hline Sample name & Details & Thickness $(\mu \mathrm{m})$ \\
\hline PCL & $\begin{array}{l}\text { Polycaprolactone electrospun thin nanofiber } \\
\text { sample }\end{array}$ & 40 \\
PCLA & $\begin{array}{l}\text { Polycaprolactone electrospun auxetic thin } \\
\text { nanofiber sample }\end{array}$ & 40 \\
PCLT & $\begin{array}{l}\text { Polycaprolactone electrospun thick nanofiber } \\
\text { sample } \\
\text { Polycaprolactone electrospun auxetic thick } \\
\text { PCLTA }\end{array}$ & 180 \\
\hline
\end{tabular}

was approximately $20 \mu \mathrm{m}$. The samples were machined at the speed of $0.3 \mathrm{~mm} / \mathrm{s}$ to prevent the fibers from sticking again because it was observed that the fibers molten by laser heat were stuck again at the feed rate faster than $0.4 \mathrm{~mm} / \mathrm{s}$. The auxetic patterned $40 \mu \mathrm{m}$ thickness thin nanofiber membrane (hereafter called as PCLA) and auxetic patterned $180 \mu \mathrm{m}$ thickness thick nanofiber membranes (hereafter called as PCLTA) were fabricated.

The abbreviations, description and thicknesses of all the samples are provided in Table 1. Photographic images of all the prepared samples have been shown in Fig. 2I.

\subsection{Physicochemical characterization of auxetic nanofiber membranes}

The morphological characterization of PCL nanofiber membrane was carried out by using scanning electron microscopy (SEM) (FEIQuanta 200 FEG, USA). The sample was coated with $5 \mathrm{~nm}$ of $\mathrm{Au} / \mathrm{Pd}$ (PECS-682) to avoid charging issue during imaging. The mechanical

I.

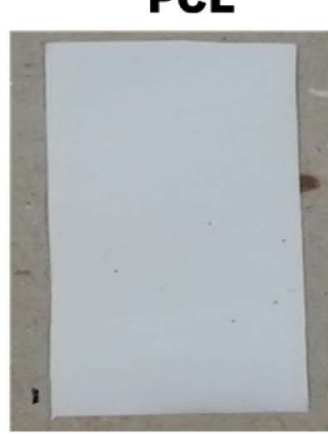

II.

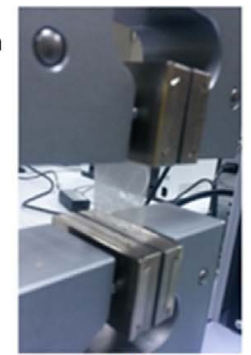

(a)

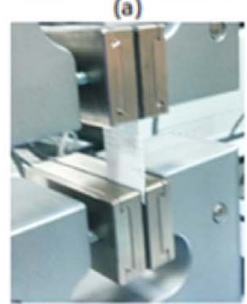

(c)
PCLA
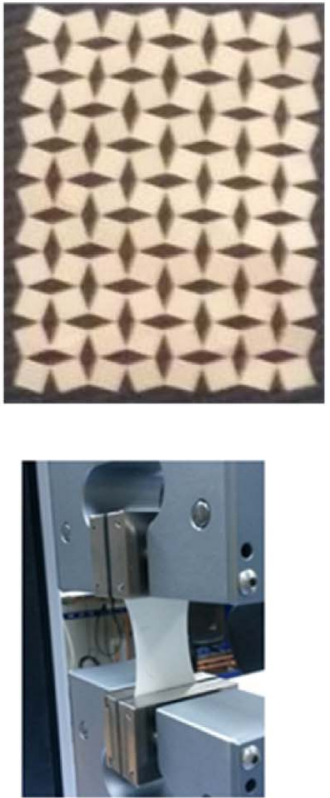

(b)

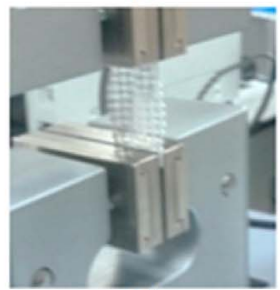

(d) properties of the fabricated PCL auxetic nanofiber membranes were characterized using uniaxial tensile testing. For PCL and PCLA samples, the rectangular sheets were cut to $35 \mathrm{~mm}$-length and $30.7 \mathrm{~mm}$-breadth such that approximately $5 \mathrm{~mm}$ grip length is assured at both ends for mechanical testing. Similarly, for PCLT and PCLTA samples, thick sheet were cut for a $37 \mathrm{~mm}$-length and $35 \mathrm{~mm}$-breadth rectangle with $7.5 \mathrm{~mm}$ grip length at both ends. A universal testing machine (Shimadzu ${ }^{\circledR}$ AGX, MAKE, Japan) with $1 \mathrm{kN}$ maximum capacity was used to perform all the test at RT at a speed of $0.5 \mathrm{~mm} / \mathrm{min}$. Non-slippery rubber fixtures were used to hold membranes and a 25-mm gauge length is allowed for the samples as shown in detail in Fig. 2II (a) to II (d), respectively. Also, the schematic of stretching behavior of auxetic samples is illustrated in Fig. 2II (e). Force and displacement were recorded for all specimens until specimens fail and break apart into two pieces. Poisson's ratio $\nu$, for all samples calculated using formula:

$\nu=-\frac{\varepsilon_{t}}{\varepsilon_{l}}$

where, $\varepsilon_{t}$ and $\varepsilon_{l}$ are transverse and longitudinal strain. The chemical group functionality of the fabricated auxetic and control PCL nanofiber membranes were analyzed using a Fourier transform infrared (FTIR) spectrometer (Nicolet is10, Thermo Scientific, USA) over the wavenumber range of $4000-400 \mathrm{~cm}^{-1}$ in ATR mode. A small piece of sample about $0.4 \mathrm{~cm}^{2}$ was placed on diamond ATR attachment and compressed with metal tip to get FTIR peaks. The thermal characterization of the samples were performed using a simultaneous TGA-DSC analyzer (600TGA, Perkin Elmer, USA) at a heating rate of $10{ }^{\circ} \mathrm{C} / \mathrm{min}$ under nitrogen atmosphere with flow rate of $100 \mathrm{ml} / \mathrm{min}$. About $5-10 \mathrm{mg}$ sample along with ceramic sample pan were used for performing the

\section{PCLT}
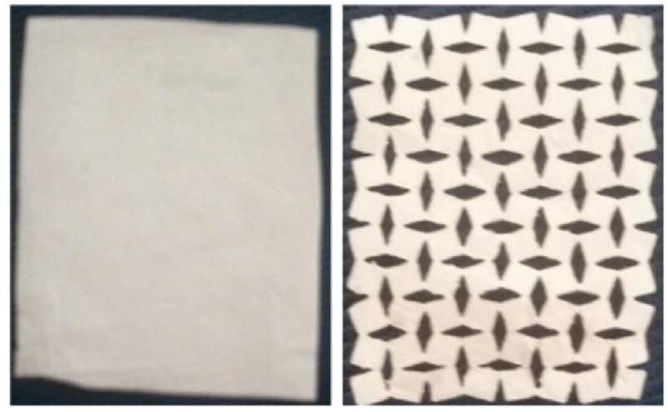

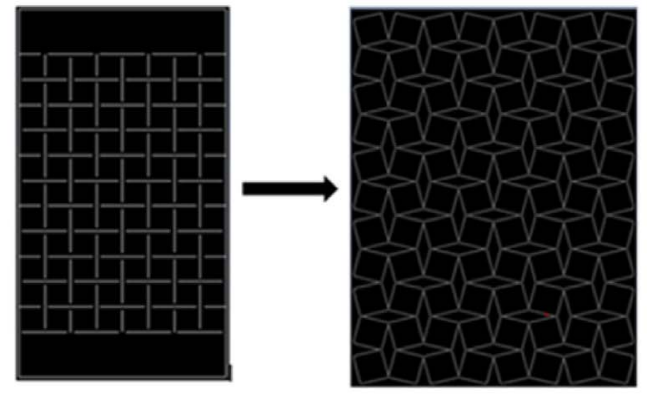

(i) (ii)

(e)

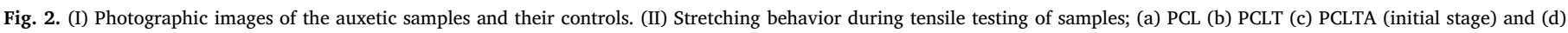
PCLTA final stage (e) schematic of deformation mechanism of sample PCLTA - compressed rotating squared pattern (i) and opened due to rotating squares (ii). 


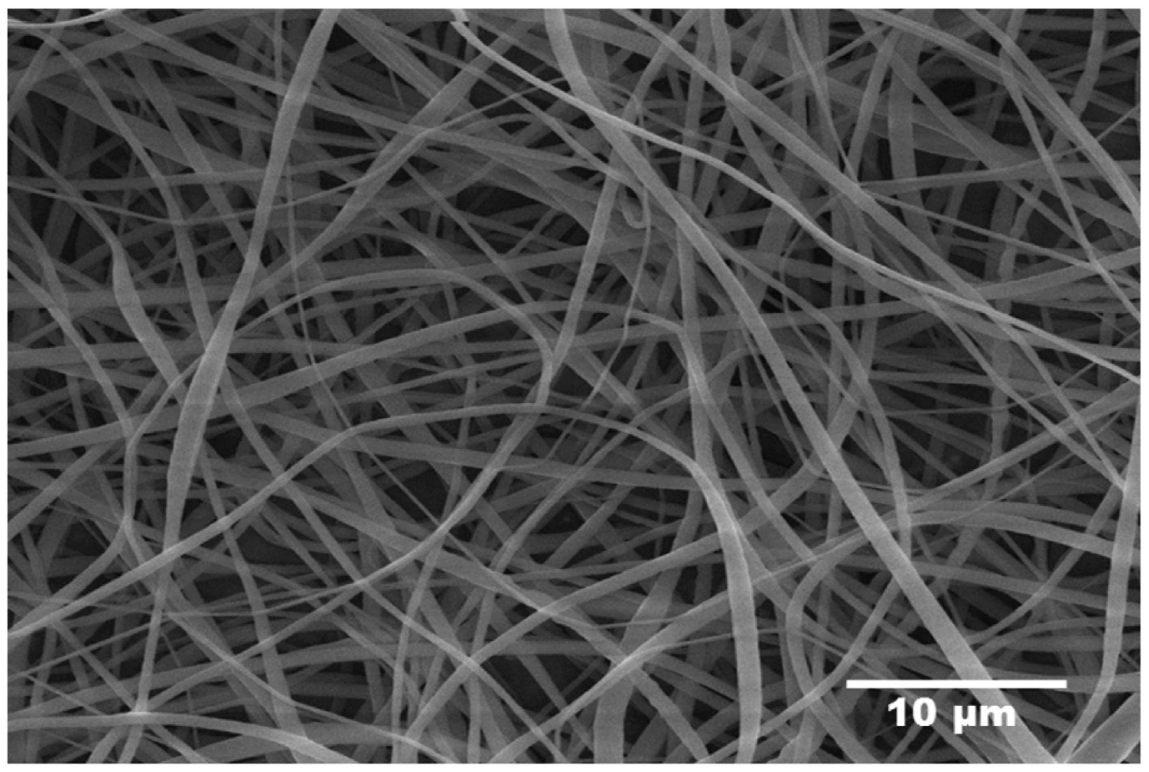

Fig. 3. SEM image of electrospun PCL nanofibers.

measurements. In order to understand the color difference due to micromachining process, the optical properties of PCL nanofiber membranes before and after tailoring auxetic patterns were determined by measuring transmittance (\%), absorbance [a.u] and reflectance (\%) at 280-1000 nm using Shimadzu UV3600 UV-Vis-NIR spectrophotometer.

\section{Results and discussion}

\subsection{SEM analysis}

SEM image of electrospun PCL nanofiber sample is shown in Fig. 3. The average fiber diameter (AFD) of nanofibers was calculated from the SEM image using ImageJ software. The diameter of about one hundred fibers was measured and the average was reported as average \pm standard deviation. The AFD for PCL sample was observed as $517 \pm 151 \mathrm{~nm}$ which confirms the formation of nanofiber structure. Since the nanofiber diameter of the developed PCL nanofibers falls within the range of the native collagen nanofibers diameter present in extracellular matrix (ECM), these electrospun nanofibers could be used as scaffolds for cell culture or various tissue engineering applications by mimicking the native ECM properties.

\subsection{Mechanical analysis}

All the four samples i.e., PCL, PCLA, PCLT and PCLTA were subjected to test for their mechanical properties using uniaxial tensile testing. By dividing the load with original cross-sectional area and dividing the extension with original gauge length, stress and strain values were computed using the formula given in Eq. (1) and depicted as a graphical representation in Fig. 4A. The stress-strain curve has been smoothened using Matlab software.

$\varepsilon_{e}=\frac{\Delta l}{l_{0}}$ and $\sigma_{e}=\frac{P}{A}$

where, $\varepsilon_{e}$ is engineering strain and $\sigma_{e}$ is the engineering stress. The details of comparative study of auxetic samples with respect to mechanical characterization and deformation mechanism are shown in Fig. 4B.

In Fig. 4, PCL sample shows a typical stress-strain curve of a ductile material such that an approximately linear elastic behavior is followed by a plastic region and failure happens following ultimate stress. The negative Poisson's value of PCLA and PCLTA samples confirms their auxetic nature. However, PCLT and PCLTA samples show a non-linear rubber-like behavior. As can be seen from the Fig. 4, PCL exhibits ultimate tensile stress of $3.3 \mathrm{MPa}$, a monotonically increasing trend and fails following the ultimate strength point. It shows approximately $15 \%$ elongation prior to failure which is in agreement with the previously published data elsewhere [2]. Moreover, PCLT samples showed an approximately linear behavior for deformations up to $33 \%$ which is followed by a nonlinear behavior until it reaches an extension of $45 \%$ followed by a linear behavior afterward up to failure. The failure in PCLT samples occurs at a $113 \%$ extension rate and it shows approximately $4 \mathrm{MPa}$ ultimate strength. Interestingly when compared to PCL samples, PCLT samples have shown higher load capacity by $12 \%$ but much more extension capability almost seven and half folds. Nevertheless, the auxetic nanofiber membranes showed much lower ultimate strength i.e., 0.33 MPa for PCLA and 0.1 MPa for PCLTA as shown in Fig. 4A (ii). In other words, their load bearing capacity is smaller than the control PCL nanofibers. It could be anticipated that the structure could have weakened by cuts made while tailoring the auxetic patterns. Consequently, the auxetic PCLA sample has lesser rigidity than the PCL sample (non-auxetic) which means that the auxetic samples could elongate much longer under the same load when compared to nonauxetic control sample. The better elongation capacity of the auxetic samples when compared with non-auxetic control PCL nanofibers could be attributed to the rotational motion ability of these auxetic structures as previously explained in Fig. 2(e). The PCLA samples showed a 150\% increase in their elongation capacity when compared to control PCL samples which confirms the more flexible nature of auxetic nanofiber membranes than the non-auxetic nanofiber membranes. Surprisingly, the total elongation capacity of the PCLA samples was found to be better than the PCLTA samples. However, the total elongation capacity of PCLTA samples were found to be reduced significantly when compared to PCLT samples, which highlights that auxeticity reduced the deformation ability for these samples. Analytical model suggests that effective Young's modulus for auxetic structure with rotating squares is inversely proportional to the thickness of squares $[35,36]$. Therefore, this reduction is an expected outcome for thick structure, however, this much reduction is attributed to the additional wrinkling effect. Wrinkling happens due to the fact that high thickness makes the square cells to bend in outward direction. Wrinkling also reduces the auxeticity of the structure because the squares are bended out of plane. To summarize, PCLA samples have showed almost ten times more elongation capacity, which confirms their more flexible nature, when compared with non-auxetic PCL samples. However, PCLTA samples have showed lower elongation capacity in comparison to non-auxetic ones due to the 

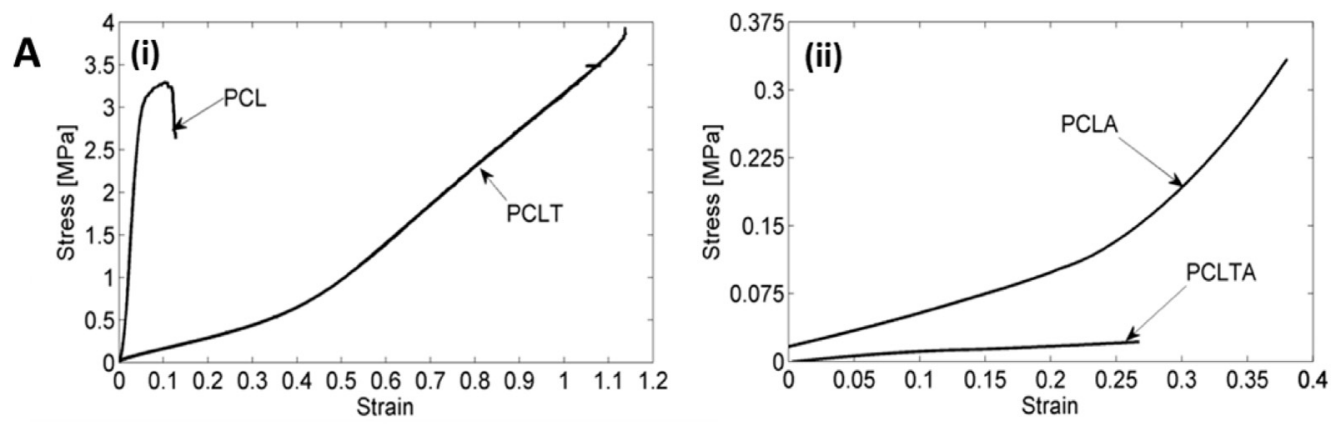

Fig. 4. (A) Stress/strain diagrams for (i) PCL and PCLT (ii) PCLA and PCLTA (iii) PCL and PCLA and (iv) PCLT and PCLTA samples. (B) Comparative analysis of elongation and ultimate strength of conventional versus auxetic PCL nanofibers.
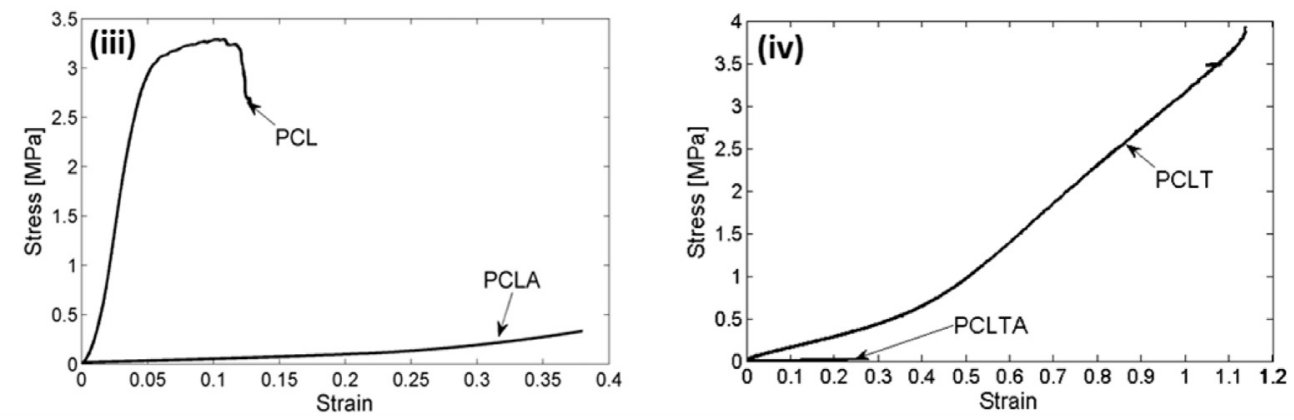

B

\begin{tabular}{|c|c|c|c|c|}
\hline \multicolumn{5}{|c|}{ Elongation and Ultimate Strength of Conventional Versus } \\
Auxetic membranes
\end{tabular}

high thickness wrinkling effect which caused reduction of the auxetic effect almost four times compared to non-auxetic form.

\subsection{FTIR analysis}

The FTIR analyses of all the four samples (PCL, PCLA, PCLT and PCLTA) were carried out to determine the chemical group functionality of the samples. Fig. 5(A) shows the FTIR spectra of PCL, PCLA, PCLT and PCLTA samples. The spectral analysis of all the samples shows the presence of typical PCL polymer absorption peaks at $2945 \mathrm{~cm}^{-1}$ and $2865 \mathrm{~cm}^{-1}$ corresponding to asymmetric $-\mathrm{CH}_{2}$ stretching and symmetric $-\mathrm{CH}_{2}$ stretching, respectively. In auxetic samples, peak at 2945 broadened to higher wavenumbers $\left(\mathrm{cm}^{-1}\right) 3003$ and 3026 for PCLA and PCLTA, respectively. In addition, strong bands such as the carbonyl stretching mode around $1720 \mathrm{~cm}^{-1}$ along with the intense sharp peak due to $\mathrm{C}=\mathrm{O}$ vibrations, can be easily identified for all samples. Additionally, in auxetic samples peaks broadening was observed which could be an effect of auxetic patterning via laser machining. The band at $1294 \mathrm{~cm}^{-1}$ is assigned to the backbone $\mathrm{C}-\mathrm{C}$ and $\mathrm{C}-\mathrm{O}$ stretching modes in the crystalline phase $[37,38]$. The peaks observed for PCL nanofibers at 2943 , and $2865 \mathrm{~cm}^{-1}$ are due to $-\mathrm{CH}_{2}$ vibrations, $-\mathrm{CH}_{2}$ bending vibrations at 1470,1417, 1396 and $1365 \mathrm{~cm}^{-1}$, and $\mathrm{C}-\mathrm{O}-\mathrm{C}$ stretching at $1238 \mathrm{~cm}^{-1}$ and $\mathrm{C}-\mathrm{O}$ vibrations at 1045 and $1106 \mathrm{~cm}^{-1}$ [39]. The auxetic samples spectra revealed the absence of peaks around $3000 \mathrm{~cm}^{-1}$ which could be due to laser machining to obtain auxetic patterns $[40,41]$. The FTIR spectrum showed all characteristic peaks of PCL, in the case of auxetic samples; however, the peak intensities were found to be lower than non-auxetic samples. In summary, auxetic tailoring did not make any significant change in the chemical functional group of the material tested.

\subsection{Thermal analysis}

The thermogram obtained by TGA analysis for the PCL, PCLA, PCLT and PCLTA samples were shown in Fig. 5(B). There was no significant weight loss observed until $190{ }^{\circ} \mathrm{C}$ for all the samples. An event of weight loss could be observed after $190^{\circ} \mathrm{C}$ in all samples due to removal of structural bound water, which can be seen between $30^{\circ} \mathrm{C}$ and $200{ }^{\circ} \mathrm{C}$ for PCL [42]. The temperature of small decomposition is determined from the curves given in Fig. 5(B) by extrapolating the curve at the peak of decomposition back to the initial weight of the PCL nanofiber. According to Fig. 5(B), PCL has thermal decomposition temperature between $270{ }^{\circ} \mathrm{C}$ and $437^{\circ} \mathrm{C}$ with maximum peak at $379{ }^{\circ} \mathrm{C}$ for PCLT and at $380^{\circ} \mathrm{C}$ for PCL, respectively. Besides, both the sample reached lowest weight percentage of remaining materials which was almost $0 \%$ at $480^{\circ} \mathrm{C}$. It was shown that, thickness of nanofiber membranes does not affected the thermal properties of the material [37]. However, as can be seen from Fig. 5(B), applying an auxetic pattern to nanofiber membranes shifted the starting temperature for thermal decomposition for both samples. In overall, it seems that the thermal stability of the nanofibers did not affect by its thickness, however, the auxetic treatment influences the thermal stability of the material tested. 
$\mathbf{A}$

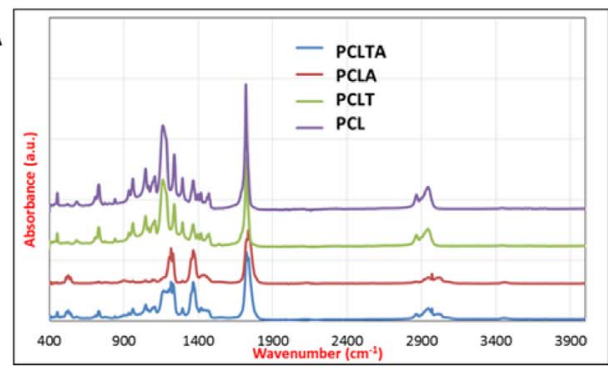

C

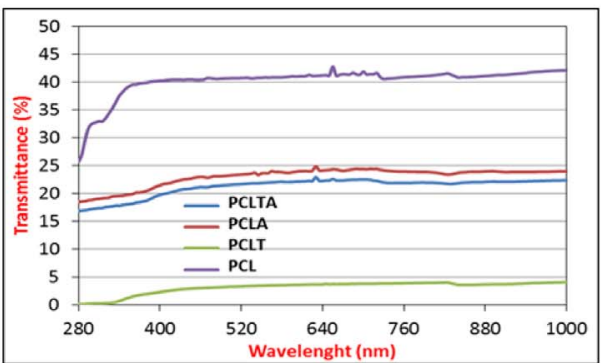

B

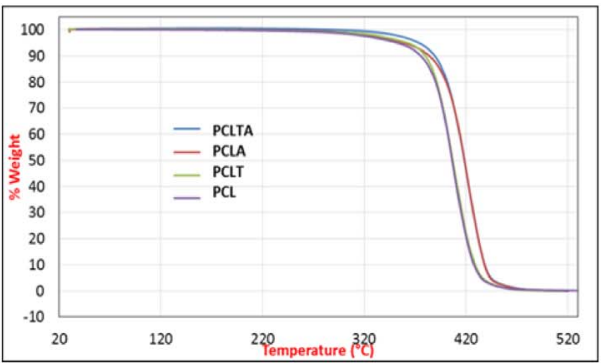

D

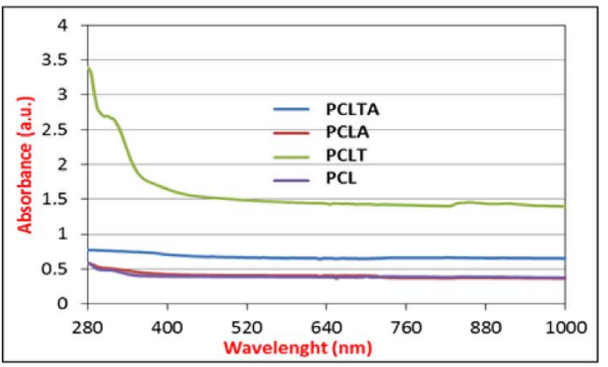

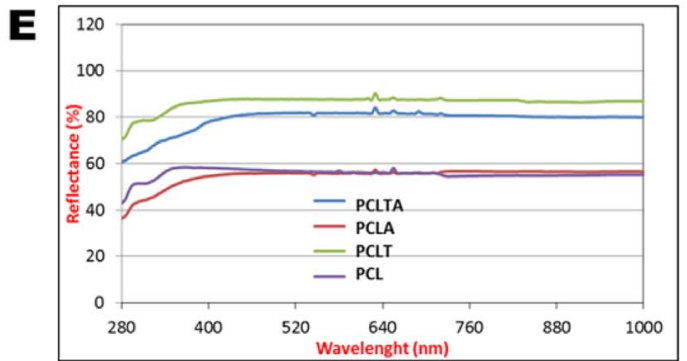

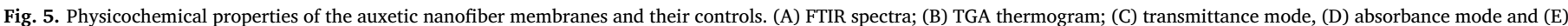
reflection mode optical properties of PCL, PCLT, PCLA and PCLTA samples.

\subsection{Optical analysis}

Optical properties of PCL nanofiber membranes with and without auxetic effect via transmittance (\%), absorbance [a.u] and reflectance (\%) measurements were also tested, keeping in mind that the data may be useful for other applications. All the nanofiber test samples are white in color and exhibited different optical measurement results due to their thicknesses and auxeticity treatment. Fig. 5(C) to Fig. 5(E) presents the optical properties (transmittance (\%), absorbance [a.u] and reflectance (\%) spectra) of PCL nanofiber membranes before and after auxetic tailoring. According to transmittance (\%) spectrum given in Fig. 5(C), a semi-transparent material (PCL sample) shows an average transmittance at $550 \mathrm{~nm}$ of around $41 \%$ transmittance due to lower thickness of the measured sample. However, thicker PCLT sample, almost opaque in nature, showed the lowest transmittance at $550 \mathrm{~nm}$ of around $3 \%$ transmittance when compared to PCL sample. After laser cutting procedure, since diamond shape opening occurred on nanofiber membranes, transparency of thick sample increased. However, in relaxed form of nanofiber membranes, these cuts formed crimps in some places. Therefore, transmittance was decreased for the thinner PCL sample. Besides, PCLT has much higher light absorption and reflectance data (around $90 \%$ in the visible region) than the PCL sample. In the visible region, when laser machining procedure was applied, absorbance of nanofiber membranes increased, while reflectance of nanofiber membranes decreased. As a result, considering nanofiber membranes with different thicknesses, auxeticity treatment via laser machining changed the transmittance of nanofiber membranes.

\section{Conclusion}

This study demonstrated that the auxetic PCL nanofiber membranes with different thicknesses can be fabricated by using electrospinning and laser micromachining. The PCLA sample showed almost ten times increase in their elongation capacity when compared to control sample of PCL, which confirms the more flexible nature of auxetic nanofiber membranes than the non-auxetic nanofiber membranes. The total elongation capacity of the PCLA sample was found to be better than the PCLTA sample. However, the total elongation capacity for PCLTA sample was found to be significantly reduced (almost six times) when compared to PCLA samples which highlights that auxeticity reduced the deformation ability of the PCLTA samples due to their increased membrane thickness. Auxetic structures bring the advantage of expansion in all directions when it is under load than the non-auxetic ones. Therefore, the auxetic PCL nanofiber membranes may serve as a potent material in various biomedical applications, in particular, tissue engineering where scaffolds with mechanical cues play a major role.

\section{Disclosures}

The authors declare no conflicts of interest.

\section{Acknowledgements}

Author acknowledges Turkish Scientific and Technical Research Council, TUBITAK, Turkey for support Project No 214M277 and CSCR, India. 


\section{References}

[1] Y.J. Park, J.K. Kim, The effect of negative Poisson's ratio polyurethane scaffolds for articular cartilage tissue engineering applications, Adv. Mater. Sci. Eng. 2013 (2013) 853289.

[2] L. Ghasemi-Mobarakeh, M.P. Prabhakaran, M. Morshed, M.H. Nasr-Esfahani, S. Ramakrishna, Electrospun poly(epsilon-caprolactone)/gelatin nanofibrous scaffolds for nerve tissue engineering, Biomaterials 29 (2008) 4532-4539.

[3] D. Kai, S.S. Liow, X.J. Loh, Biodegradable polymers for electrospinning: towards biomedical applications, Mater. Sci. Eng. C 45 (2015) 659-670.

[4] X.J. Loh, P. Peh, S. Liao, C. Sng, J. Li, Controlled drug release from biodegradable thermoresponsive physical hydrogel nanofibers, J. Control. Release 143 (2010) 175-182.

[5] D. Kai, M.J. Tan, M.P. Prabhakaran, B.Q.Y. Chan, S.S. Liow, S. Ramakrishna, X.J. Loh, Biocompatible electrically conductive nanofibers from inorganic-organic shape memory polymers, Colloids Surf. B: Biointerfaces 148 (2016) 557-565.

[6] D. Kai, M. Prabhakaran, B. Chan, S. Liow, S. Ramakrishna, F. Xu, X. Loh, Elastic poly( $\varepsilon$-caprolactone)-polydimethylsiloxane copolymer fibers with shape memory effect for bone tissue engineering, Biomed. Mater. 11 (2016) 015007.

[7] C.J. Chirayil, L. Mathew, S. Thomas, Review of recent research in nano cellulose preparation from different lignocellulosic fibers, Rev. Adv. Mater. Sci. 37 (2014) 20-28.

[8] J. Hu, D. Kai, H. Ye, L. Tian, X. Ding, S. Ramakrishna, X.J. Loh, Electrospinning of poly(glycerol sebacate)-based nanofibers for nerve tissue engineering, Mater. Sci. Eng. C 70 (2017) 1089-1094.

[9] D. Kai, S. Jiang, Z.W. Low, X.J. Loh, Engineering highly stretchable lignin-based electrospun nanofibers for potential biomedical applications, J. Mater. Chem. B 3 (2015) 6194-6204.

[10] R. Lakshminarayanan, R. Sridhar, X.J. Loh, M. Nandhakumar, V.A. Barathi, M. Kalaipriya, J.L. Kwan, S.P. Liu, R.W. Beuerman, S. Ramakrishna, Interaction of gelatin with polyenes modulates antifungal activity and biocompatibility of electrospun fiber mats, Int. J. Nanomedicine 9 (2014) 2439-2458.

[11] D. Kai, W. Ren, L. Tian, P.L. Chee, Y. Liu, S. Ramakrishna, X.J. Loh, Engineering poly(lactide)-lignin nanofibers with antioxidant activity for biomedical application, ACS Sustain. Chem. Eng. 4 (2016) 5268-5276.

[12] M.A.K. Liebschner, M.A. Wettergreen, Optimization of bone scaffold engineering for load bearing applications, Top. Tissue Eng. (2003) 1-39.

[13] G.M. Cunniffe, F.J. O'Brien, Collagen scaffolds for orthopedic regenerative medicine, JOM 63 (2011) 66-73.

[14] P. Soman, J.W. Lee, A. Phadke, S. Varghese, S. Chen, Spatial tuning of negative and positive Poisson's ratio in a multi-layer scaffold, Acta Biomater. 8 (2012) 2587-2594.

[15] A. Bezazi, F. Scarpa, Mechanical behaviour of conventional and negative Poisson's ratio thermoplastic polyurethane foams under compressive cyclic loading, Int. J. Fatigue 29 (2007) 922-930.

[16] J. Lisiecki, S. Klysz, T. Blazejewicz, G. Gmurczyk, P. Reymer, Tomographic examination of auxetic polyurethane foam structures, Phys. Status Solidi Basic Res. 251 (2014) 314-320.

[17] A. Alderson, K.L. Alderson, S.A. McDonald, B. Mottershead, S. Nazare, P.J. Withers, Y.T. Yao, Piezomorphic materials, Macromol. Mater. Eng. 298 (2013) 318-327.

[18] F. Pierron, S.A. McDonald, D. Hollis, J. Fu, P.J. Withers, A. Alderson, Comparison of the mechanical behaviour of standard and auxetic foams by x-ray computed tomography and digital volume correlation, Strain 49 (2013) 467-482.

[19] R.S. Lakes, Foam structures with a negative Poisson's ratio, Science 235 (1987) 1038-1040.

[20] F. Quadrini, D. Bellisario, L. Ciampoli, G. Costanza, L. Santo, Auxetic epoxy foams produced by solid state foaming, J. Cell. Plast. 52 (2016) 441-454.

[21] Y. Li, C. Zeng, Room-temperature, near-instantaneous fabrication of auxetic materials with constant Poisson's ratio over large deformation, Adv. Mater. 28 (2016) 2822-2826.

[22] D.R. Veronda, R.A. Westmann, Mechanical characterization of skin-finite deformations, J. Biomech. 3 (1970) 111-122.

[23] L.M. Frolich, M. LaBarbera, W.P. Stevens, Poisson's ratio of a crossed fibre sheath: the skin of aquatic salamanders, J. Zool. 232 (1994) 231-252.

[24] J.L. Williams, J.L. Lewis, Properties and an anisotropic model of cancellous bone from the proximal tibial epiphysis, J. Biomech. Eng. 104 (1982) 50-56.

[25] L. Liulan, H. Qingxi, H. Xianxu, X. Gaochun, Design and fabrication of bone tissue engineering scaffolds via rapid prototyping and CAD, J. Rare Earths 25 (2007) 379-383.

[26] S.K. Bhullar, Characterization of auxetic polyurethanes foam for biomedical implants, E-Polymers 14 (2014) 441-447.

[27] J. Ko, S.K. Bhullar, Y. Cho, P.C. Lee, M. Byung-Guk Jun, Design and fabrication of auxetic stretchable force sensor for hand rehabilitation, Smart Mater. Struct. 24 (2015) 75027.

[28] S.K. Bhullar, N.L. Lala, S. Ramkrishna, Smart biomaterials - a review, Rev. Adv. Mater. Sci. 40 (2015) 303-314.

[29] S.K. Bhullar, J. Ko, Y. Cho, M.B.G. Jun, Fabrication and characterization of nonwoven auxetic polymer stent, Polym.-Plast. Technol. Eng. 54 (2015) 1553-1559.

[30] Y. Pan, S. Dong, Y. Hao, T. Chu, C. Li, Z. Zhang, Y. Zhou, Demineralized bone matrix gelatin as scaffold for tissue engineering, Afr. J. Microbiol. Res. 4 (2010) 865-870.

[31] M.N. Ali, I.U. Rehman, Auxetic polyurethane stents and stent-grafts for the palliative treatment of squamous cell carcinomas of the proximal and mid oesophagus: a novel fabrication route, J. Manuf. Syst. 37 (2015) 375-395.

[32] L. Zhang, T.J. Webster, Nanotechnology and nanomaterials: promises for improved tissue regeneration, Nano Today 4 (2009) 66-80.

[33] M. Ramalingam, M.F. Young, V. Thomas, L. Sun, L.C. Chow, C.K. Tison, K. Chatterjee, W.C. Miles, C.G. Simon, Nanofiber scaffold gradients for interfacial tissue engineering, J. Biomater. Appl. 27 (2013) 695-705.

[34] Y. Wang, D.J. Blasioli, H.J. Kim, H.S. Kim, D.L. Kaplan, Cartilage tissue engineering with silk scaffolds and human articular chondrocytes, Biomaterials 27 (2006) 4434-4442.

[35] D. Attard, E. Manicaro, R. Gatt, J.N. Grima, On the properties of auxetic rotating stretching squares, Phys. Status Solidi Basic Res. 246 (2009) 2045-2054.

[36] T.-C. Lim, Auxetic Materials and Structures, 1st ed., Springer Singapore, 2015, http://www.springer.com/in/book/9789812872746.

[37] Y. Lai, G. Veser, ZnO@ZSM-5 Nanostructured Core-Shell Catalysts for Methane Dehydroaromatization to Benzene, 2013 AIChE Annu. Meet. 2013 https://www. aiche.org/conferences/aiche-annual-meeting/2013/proceeding/paper/263eznozsm-5-nanostructured-core-shell-catalysts-methane-dehydroaromatizationbenzene-1.

[38] H. Zhou, Electrospun Fibers from both Solution and Melt: Processing, Structure and Property, Cornell University, 2007, https://core.ac.uk/download/pdf/4904389. pdf.

[39] V.Y. Chakrapani, A. Gnanamani, V.R. Giridev, M. Madhusoothanan, G. Sekaran, Electrospinning of type I collagen and PCL nanofibers using acetic acid, J. Appl. Polym. Sci. 125 (2012) 3221-3227.

[40] M.M. Coleman, D.F. Varnell, Fourier-transform infrared studies of polymer blends 3 poly(beta -propiolactone)-poly(vinyl chloride) system, J. Polym. Sci. Part A-2, Polym. Phys. 18 (1980) 1403-1412.

[41] J.E. Oliveira, L.H.C. Mattoso, W.J. Orts, E.S. Medeiros, Structural and morphological characterization of micro and nanofibers produced by electrospinning and solution blow spinning: a comparative study, Adv. Mater. Sci. Eng. 2013 (2013) 409572.

[42] E.C. Chen, T.M. Wu, Isothermal crystallization kinetics and thermal behavior of poly(?-caprolactone)/multi-walled carbon nanotube composites, Polym. Degrad. Stab. 92 (2007) 1009-1015. 